\title{
Blood Flow Velocity Changes in the Middle Cerebral Artery Induced by Driving Fatigue
}

\author{
Hong Wang, Xiaomei Chi, Yajing Yan and Ningning Zhang \\ Northeastern University, 110004 Shenyang, China \\ hongwang@mail.neu.edu.cn
}

Keywords: Driving fatigue, TCD, Cerebral blood flow velocity, Head massage

\begin{abstract}
The cerebral blood flow velocity (CBFV) of middle cerebral artery (MCA) was detected during the fatigue driving using Transcranial Doppler. The CBFV was also analyzed after the fatigue driving by different means of relaxation to alleviate brain fatigue. The results show that the CBFV in MCA is reduced by driving fatigue.
\end{abstract}

\section{Introduction}

Transcranial Doppler (TCD) was first described by Aaslid et al. in 1982 [1]. Because of a high temporal resolution TCD has become a widely used method to determine cerebral blood flow velocity (CBFV) of the basal cerebral arteries during specific stimulation conditions [2-5]. Frauenfelder et al. found that specific CBFV patterns support the idea of different cognitive challenges for planning and control between easy and difficult conditions [6]. Schnittger et al. reported that the differential blood flow changes in the two middle cerebral arteries (MCA) as a function of attention to the visual stimulus [7].

In USA there were 42,643 persons killed and 2,889,000 persons injured in traffic crashes in 2003 [8]. One of the causes for motor vehicle crashes is the effect of fatigue driving [9-10]. But how is the CBFV during the driving fatigue? Could the CBFV be changed using the head massage after driving fatigue? In this paper blood flow velocity changes in MCA induced by driving fatigue were researched.

\section{Materials and methods}

Eighteen right-handed healthy volunteers (range 20-30 years) participated in the experiment. Tran cranial Doppler measurements were performed with a COMPANION III TCD instrument. The cerebral blood velocity was measured from the right MCA using TCD. A 2-MHz pulsed wave transducer was placed over the temporal window and fixed with a headband. Peak mean CBFV of MCA (Fig.1) that represents the mean CBFV of the envelope curve was assessed in all examined vessels. The head massages were performed with a massager (breo $\left.{ }^{\circledR}\right)$. The on-board measurements were conducted in the experimental car.

Continuous variables are expressed as mean $\pm \mathrm{SD}$. We used the paired Student t-test to compare peak mean CBFV of MCAs between control group and the experiment group. All tests were 2-sided, and a value of $\mathrm{p}<0.05$ was considered statistically significant.

The subjects were divided into three groups (Fig.2), the control group, experimental group 1 (15min head massage after driving) and experimental group 2 (15 min natural break after driving). The experiments are as follows. In the beginning of the experiment, the cerebral blood velocities were measured from the right MCA of all subjects. After that, the subjects in experimental group 1 and 2 drove the experimental car for $1 \mathrm{~h}$. Then, the cerebral blood velocities were measured from the right MCA of all subjects. Subjects in experimental group 2 took $15 \mathrm{~min}$ natural break after driving. Subjects in experimental group 1 were performed with the 15 min head massager. At 85 min after the beginning of the experiment, the cerebral blood velocities were measured from the right MCA of all subjects. 


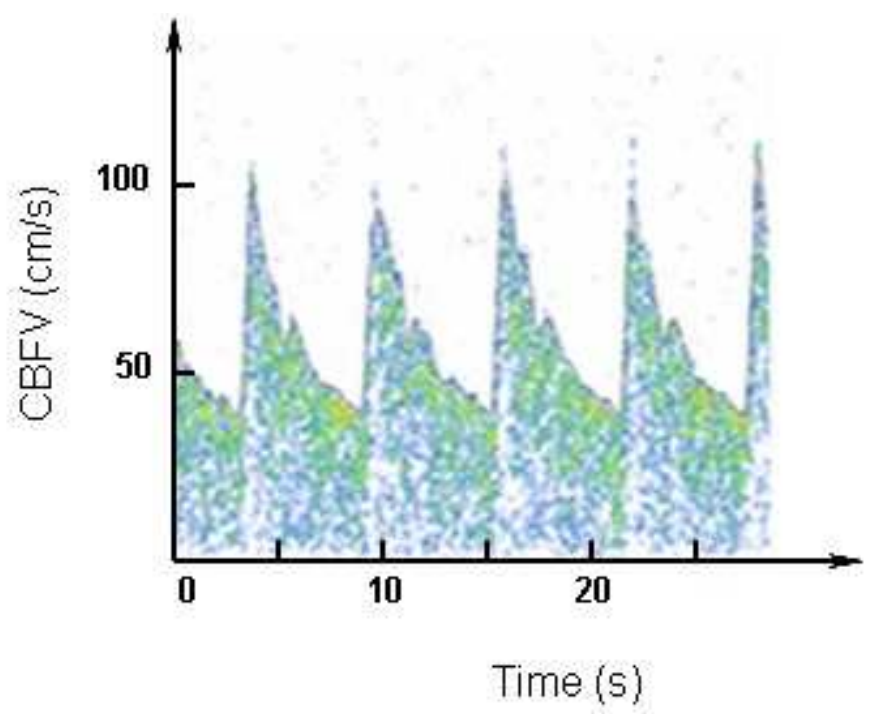

Fig. 1. CBFV of MCA
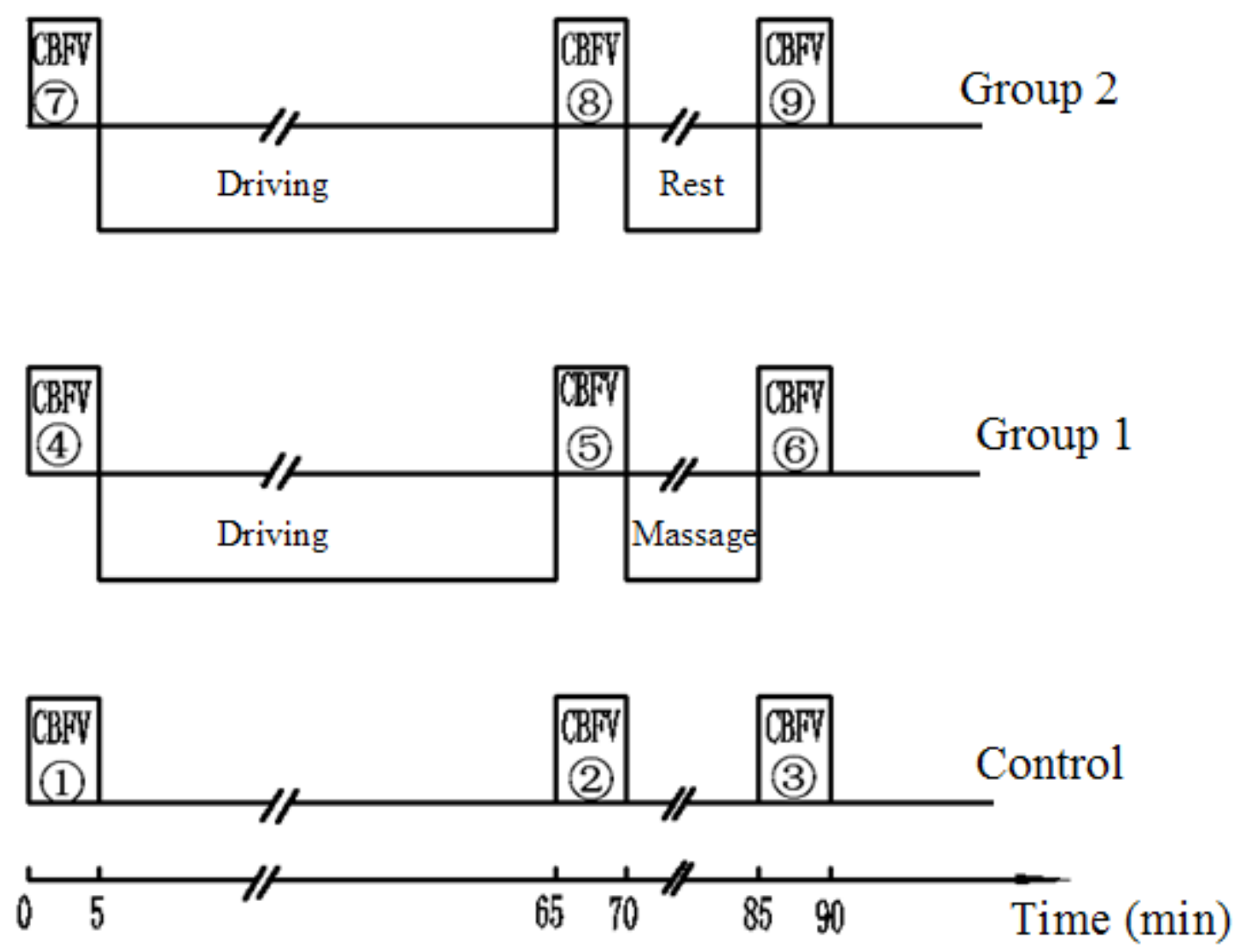

Fig. 2. Experimental program

\section{Results}

The results show that cerebral blood flow velocities (CBFVs) of the right MCA after 1h driving are less than the control group (Fig.3, $\mathrm{P}<0$. 01), i.e. the blood flow velocities in MCA are reduced after $1 \mathrm{~h}$ driving. It means that fatigue driving could bring about the decrease of the CBFV. After 15min natural break, the CBFV began to recover (Fig.4). The recovering effect through $15 \mathrm{~min}$ head massage is better than through $15 \mathrm{~min}$ natural break (Fig.4). There is the significant different $(p<0.05)$ between the CBFV changes induced by $15 \mathrm{~min}$ head massage after the driving and just after the driving. 


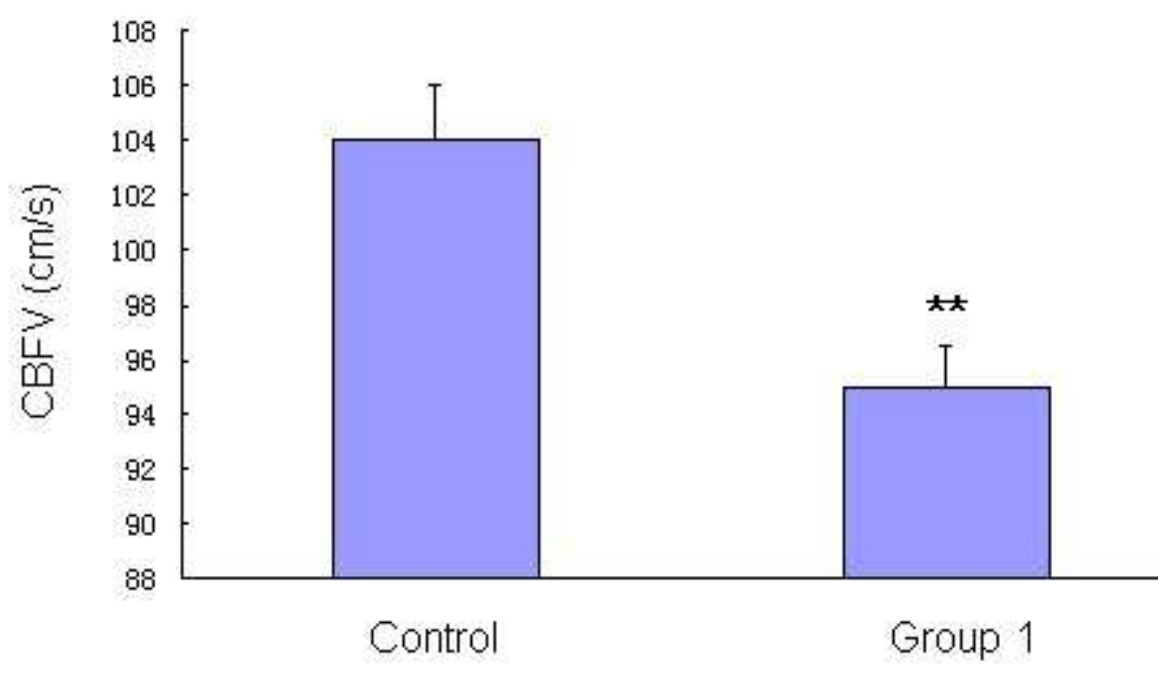

Fig. 3. Decrease of CBFV after $1 \mathrm{~h}$ driving $(* * \mathrm{p}<0.01)$

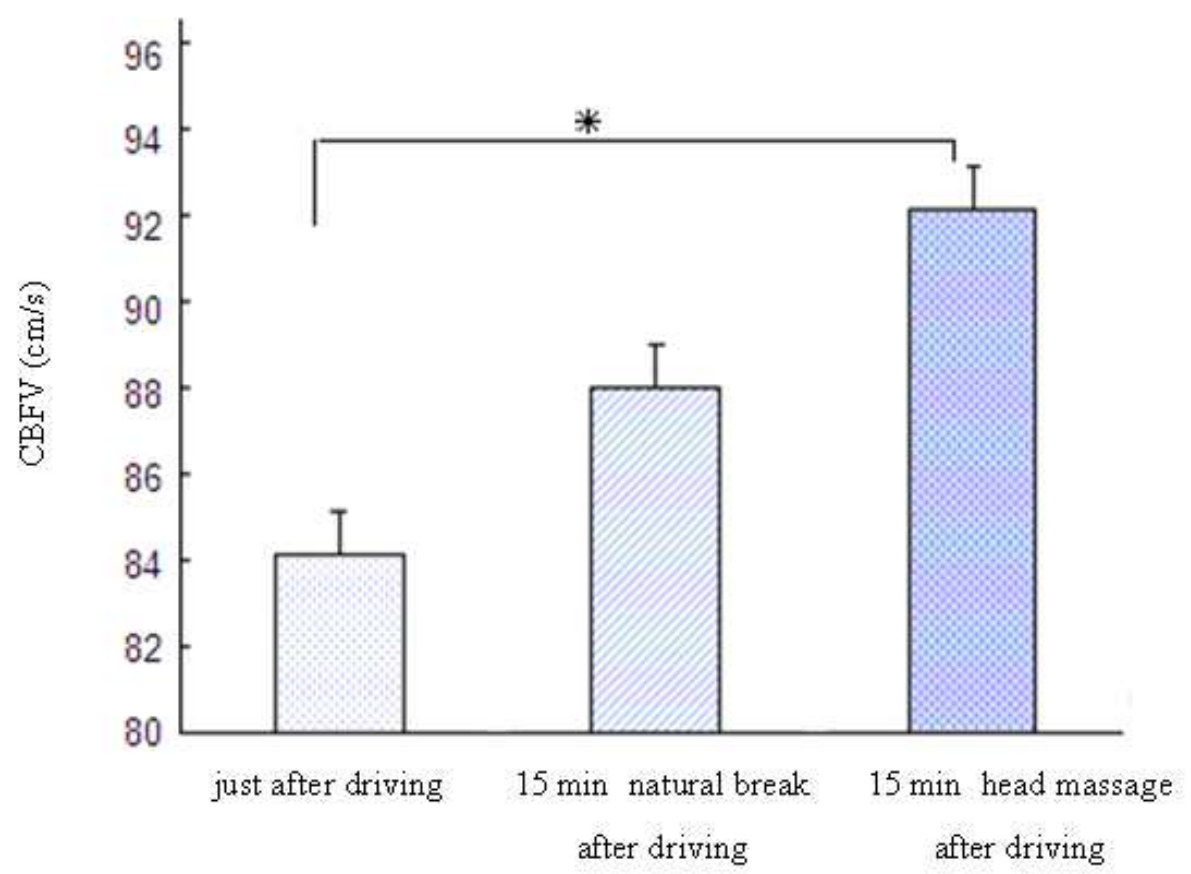

Fig. 4. CBFV changing $(* \mathrm{p}<0.05)$

\section{Discussion}

In this paper, the effects of fatigue driving on cerebral blood flows have been showed. The cerebral blood flows of middle cerebral artery were detected during the fatigue driving by the Transcranial Doppler in real time, and compared to analyze the effects of fatigue driving on blood flows velocity. The cerebral blood flows were also detected after the fatigue driving by different means of relaxation to alleviate brain fatigue. The results show that the CBFV in MCA is reduced by fatigue driving. After 15 min natural break after the driving the CBFV began to recover. The recovering effect by $15 \mathrm{~min}$ head massage after the driving is better than the effect after $15 \mathrm{~min}$ natural break. There is the significant different $(p<0.05)$ between the CBFV changes induced by $15 \mathrm{~min}$ head massage after the driving and just after the driving. 
It has been reported that the oxygenation delivery of brain tissue is associated with the cerebral blood flow velocities [11-13]. The decrease of CBFV in MCA means that the oxygenation delivery of brain tissue could come down after $1 \mathrm{~h}$ driving. If the oxygenation of brain tissue is insufficient, subjective activation could be reduced. It could easily lead to motor vehicle crashes. Through $15 \mathrm{~min}$ natural break after driving the CBFV could be gradually recovered with the recovering of the oxygenation delivery in brain tissue. The head massage is a method of brain rehabilitation [14].The head massage could speed up the rehabilitation of the oxygenation delivery in brain tissue. That means that CBFV could be faster recovered by the head massage after driving fatigue. The CBFV of MCA using TCD may be a physiological index for checking the level of the driving fatigue.

\section{Acknowledgment}

This work was supported by National Science Foundation of China (61071057), Fundamental Research Funds for the Central Universities of China (N100603003).

\section{References}

[1] R. Aaslid, T.M. Markwalder, H. Nornes, Noninvasive transcranial doppler ultrasound recording of flow velocity in basal cerebral arteries, J. Neurosurg. 57(1982) 769- 774.

[2] J.E. Peltonen, J.M. Kowalchuk, D.H. Paterson, D.S. DeLorey, G.R. duManoir, R.J. Petrella, J.K. Shoemaker, Cerebral and muscle tissue oxygenation in acute hypoxic ventilatory response test, Respiratory Physiology \& Neurobiology. 155 (2007) 71-81.

[3] H.H. Van, P. Poommipanit, M. Shalaby, R. Gevorgyan, C.H. Tseng, J. Tobis, Sensitivity of transcranial doppler versus intracardiac echocardiography in the detection of right-to-left shunt, Cardiovascular Imaging. 4 (2010) 343-348.

[4] R.G. Wise, K. Ide, M.J. Poulin, I. Tracey, Resting fluctuations in arterial carbon dioxide induce significant low frequency variations in BOLD signal, NeuroImage. 21 (2004) 1652- 1664.

[5] G. Vingerhoets, E. Luppens, Cerebral blood flow velocity changes during dichotic listening with directed or divided attention: a transcranial doppler ultrasonography study, Neuropsychologia. 39 (2001) 1105-1111.

[6] B.A. Frauenfelder, D. Schuepbach, R.W. Baumgartner, D. Hell, Specific alterations of cerebral hemodynamics during a planning task: a transcranial Doppler sonography study, NeuroImage. 22 (2004) 1223-1230.

[7] C. Schnittger, S. Johannes, A. Arnavaz, T.F. Munte, Blood flow velocity changes in the middle cerebral artery induced by processing of hierarchical visual stimuli, Neuropsychologia. 8 (1997) 1181-1184.

[8] N. Rhodes, D. Brown, A. Edison, Approaches to understanding young driver risk taking, J. Safety Research-Traffic Records Forum proceedings. 36 (2005) 497-499.

[9] G. Yang, Y.Z. Lin, B. Prabir, A driver fatigue recognition model based on information fusion and dynamic Bayesian network, Information Sciences. 180 (2010) 1942-1954.

[10] J. Wang, W. Xu, Y. Gong, Real-time driving danger-level prediction, Engineering Applications of Artificial Intelligence. 23 (2010) 1247-1254.

[11] P.J. Cohen, S.C. Alexander, T.C. Smith, M. Reivich, H. Wollman, Effects of hypoxia and normocarbia on cerebral blood flow and metabolism in conscious man, J. Appl. Physiol. 23 (1067) 183-189.

[12] J.B. Jensen, B. Sperling, J.W. Severinghaus, N.A. Lassen, Augmented hypoxic cerebral vasodilation in men during 5 days at 3,810m altitude, J. Appl. Physiol. 80 (1996) 1214-1218.

[13] J.C. Kolb, P.N. Ainslie, K. Ide, M.J. Poulin, Protocol to measure acute cerebrovascular and ventilatory responses to isocapnic hypoxia in humans, Respir. Physiol. Neurobiol. 141 (2004) 191-199.

[14] S. Fritz, L. Chaitow, G.M. Hymel, Massage Application Assessment for Physical Healing and Rehabilitation, in: Clinical Massage in the Healthcare Setting, Elsevier Inc., Mosby, 2001, pp.248-313. 